\title{
Optical Coherence Tomography Fast versus Regular Macular Thickness Mapping in Diabetic Retinopathy
}

\author{
Lala Ceklic $^{\mathrm{a}}$ Noémi Maár $^{\mathrm{b}} \quad$ Aljoscha S. Neubauer ${ }^{\mathrm{c}}$ \\ a Department of Ophthalmology, Clinical Center of Eastern Sarajevo, RS-Bosnia and Herzegovina University of \\ Sarajevo, Sarajevo, RS-Bosnia; ${ }^{b}$ Department of Ophthalmology and Optometry, Medical University of Vienna, \\ Vienna, Austria; 'Department of Ophthalmology, Ludwig Maximilians University, Munich, Germany
}

\section{Key Words}

Retinopathy · Optical coherence tomography $\cdot$ Macular edema $\cdot$ Imaging $\cdot$ Diabetes

\begin{abstract}
Objective: The purpose of the study was to investigate if absolute values and reproducibility of thickness maps obtained from 2 optical coherence tomography (OCT) scanning protocols, regular high-resolution and fast low-density mode, differ in patients with diabetic macular edema. Methods: A total of 26 consecutive patients undergoing fluorescein angiography and Stratus OCT scanning for the evaluation of diabetic macular edema at the Departments of Ophthalmology in Munich and Vienna were included. Results: Retinal thickness of the central field of the thickness map measured by fast retinal thickness protocol was $287 \pm 97$ and $290 \pm$ $113 \mu \mathrm{m}$ by the regular protocol. This difference as well as that for all other fields was not statistically significant. Three times repeated measurements applying both OCT scanning modes in 10 patients yielded very good intrasession correlation coefficients between 0.70 and 0.99 , with corresponding intrasession standard deviations ranging between 6 and 16 $\mu \mathrm{m}$. The fast mode yielded slightly less reproducible values than the regular mode. Visual acuity did not influence the results. Conclusion: In practice both scanning modes can be
\end{abstract}

interchanged and absolute values can be compared directly. Best reproducibility is obtained with higher sampling density even in patients with reduced visual acuity due to diabetic macular edema.

Copyright $\odot 2008$ S. Karger AG, Basel

\section{Introduction}

Diabetic retinopathy is a leading cause of severe visual impairment and blindness in the developed countries. The incidence and prevalence are high and continue to increase [1]: it is estimated that currently alone in the USA 10.2 million adults aged 40 years or older have diabetes mellitus. Besides proliferative disease diabetic macular edema is the major vision-threatening retinal change [2]. For its treatment the Early Treatment Diabetic Retinopathy Study (ETDRS) has defined distinct criteria and shown the effectiveness of focal laser coagulation $[3,4]$. In addition several new medical treatment options have become available recently, such as anti-VEGF agents or PKC inhibitors $[5,6]$. Therefore appropriate and timely detection of macular edema has increasing importance in order to offer patients the best possible treatment and prevent vision loss.

Besides stereoscopic, biomicroscopic fundus examination [7], over the last years new objective methods were

\section{KARGER}

Fax +41613061234 E-Mail karger@karger.ch www.karger.com
(C) 2008 S. Karger AG, Basel 0030-3747/08/0405-0235\$24.50/0

Accessible online at:

www.karger.com/ore
Aljoscha S. Neubauer, MD, MBA

Department of Ophthalmology, Ludwig Maximilians University Munich

Mathildenstrasse 8, DE-80336 Munich (Germany)

Tel. +49 895160 5160, Fax +49 913151603051

E-Mail aljoscha.neubauer@med.uni-muenchen.de 
established to quantify the retinal thickness of the posterior pole. Optical coherence tomography (OCT) is most frequently applied and allows objective measurements of macular thickness. With several scans it can generate detailed retinal thickness maps covering the posterior pole. The instrument allows to reliably detect such small increases of retinal thickness of $<10 \mu \mathrm{m}$ [8-10], while stereoscopic fundus examination usually only detects retinal thickening of approximately $100 \mu \mathrm{m}$ [11]. OCT therefore is believed to be superior to clinical examination for detecting small amounts of retinal thickening in diabetic macular edema $[12,13]$. However, the currently most frequently applied OCT, the Stratus OCT (Carl Zeiss Meditec, Jena, Germany), offers 2 modes for measuring macular thickness maps: a regular high-resolution mode and a fast mode. Although the resolution in the $x-y$ plane and scanning times differ significantly, both modes are applied and interchanged in clinical practice, to date without proper validation. Knowledge about any possible differences between the 2 scanning modes is especially of importance for cases where only the fast mode gives results due to patient disability or compliance. Therefore, the aim of this study was to investigate if absolute values and reproducibility of these 2 OCT scanning modes differ in patients with diabetic macular edema.

\section{Methods}

\section{Patients}

A total of 26 consecutive patients undergoing fluorescein angiography and Stratus OCT scanning for the evaluation of diabetic maculopathy at the Departments of Ophthalmology in $\mathrm{Mu}$ nich and Vienna were included in this study. From each patient only 1 eye was randomly selected for further analysis. Inclusion criteria were acceptable metabolic control defined by a hemoglobin $A_{1 c}$ of $<8 \%$, acceptable control of blood pressure $(<140 / 90 \mathrm{~mm}$ $\mathrm{Hg}$ ) and some degree of clinically significant diabetic macular edema according to the ETDRS criteria [14] on clinical stereoscopic slit lamp examination. Both proliferative and nonproliferative diabetic retinopathy were included and no previous surgery to the posterior segment was allowed, while mild focal laser therapy was. Further exclusion criteria were neovascular glaucoma, no visual function (stability of fixation was no inclusion or exclusion criteria), vitreous hemorrhage and all other eye diseases, especially those involving the macula. A full ophthalmological examination including a dilated ( $1 \%$ tropicamide) stereoscopic fundus exam (biomicroscope, 78-dpt lens) was performed by retinal specialists (A.S.N., N.M.). In all patients both fluorescein angiography and OCT were carried out after informed consent. All research was conducted in accordance with institutional guidelines and board approval and conformed to the tenets of the World Medical Association Declaration of Helsinki.
Table 1. Patient characteristics

\begin{tabular}{|c|c|c|}
\hline & Distribution & $\begin{array}{l}\text { Statistical } \\
\text { significance } \\
\mathrm{p}\end{array}$ \\
\hline Gender & $\begin{array}{l}18 \text { male }(69) \\
8 \text { female }(31)\end{array}$ & 0.07 \\
\hline Eye & $\begin{array}{l}10 \text { right (38) } \\
16 \text { left }(62)\end{array}$ & 0.33 \\
\hline Age, years & $64 \pm 20[24-77]$ & - \\
\hline Diabetes type & $\begin{array}{c}9 \text { IDDM (35) } \\
17 \text { NIDDM (65) }\end{array}$ & 0.17 \\
\hline $\begin{array}{l}\text { Duration of diabetes, } \\
\text { years }\end{array}$ & $14 \pm 14[1-26]$ & - \\
\hline Hemoglobin $A_{1 c}, \%$ & $7.0 \pm 0.6[5.7-7.7]$ & - \\
\hline Retinopathy level & $\begin{array}{l}2 \text { mild NPDR }(8) \\
13 \text { moderate NPDR (50) } \\
5 \text { severe NPDR (19) } \\
6 \text { PDR }(23)\end{array}$ & $0.02^{*}$ \\
\hline $\begin{array}{l}\text { Visual acuity } \\
\quad \text { (logMAR of Snellen) }\end{array}$ & $0.40 \pm 0.41[0-2.0]$ & - \\
\hline
\end{tabular}

Given are the median with interquartile range in square brackets or number of patients with percentages in parentheses. ${ }^{*} \mathrm{p}=$ 0.02: significant difference. IDDM $=$ Insulin-dependent diabetes mellitus; NIDDM = non-insulin-dependent diabetes mellitus; $\mathrm{NPDR}=$ nonproliferative diabetic retinopathy; PDR = proliferative diabetic retinopathy.

\section{OCT Measurements}

OCT (Carl Zeiss Meditec) was performed with a commercially available unit of the Stratus OCT-III. The principles of OCT have been described in detail elsewhere $[15,16]$. In this study software version 4.04 was applied to measure retinal thickness applying a scan length of $6.0 \mathrm{~mm}$. Internal fixation of the OCT was used. Six radial scans every $30^{\circ}$ were performed and the instrument's built-in, automatic mapping algorithm was applied to automatically calculate the retinal maps. Retinal thickness in the 9 ETDRS fields was analyzed. In each patient, 2 maps were generated: 1 consisting of scans in the regular scanning mode applying 512 A scans and 1.28 s per scan and 1 with the fast macular scanning applying less dense $128 \mathrm{~A}$ scans in $0.32 \mathrm{~s}$ per scan. The 2 scan protocols were performed in random order. Additionally, in 10 randomly selected patients the 2 mapping protocols (fast and regular) were applied 3 times in random order to determine the intrasession reproducibility.

\section{Statistics}

Data were collected and analyzed using SPSS 13.0 for Windows (SPSS Inc., Chicago, Ill., USA). On all statistical tests $\mathrm{p}<$ 0.05 was considered significant. Multi- and univariate analyses were applied. Paired testing and nonparametrical methods (e.g. Spearman correlation) were chosen where appropriate, which was especially the case for the not normally distributed OCT thickness data. 


\section{Results}

\section{Patients}

The demographic characteristics of the 26 patients included are summarized in table 1 . Visual acuity ranged from $\log$ MAR $0-2$ with a median of 0.40 , corresponding to Snellen 20/50 (range 20/2,000-20/20).

\section{OCT Macular Map Comparison}

The median retinal thickness of the central field measured by the fast retinal thickness protocol was $287 \pm 97$ and $290 \pm 113 \mu \mathrm{m}$ (median \pm interquartile range) by the regular macular retinal thickness protocol. This difference as well as that for all other fields was not statistically significant. A sample patient illustrating the good concordance of the results is shown in figure 1 . The details of the measurements are listed in table 2 . The correlation coefficients of the values obtained by the 2 scanning modes were very good, ranging between 0.72 and 0.95 .

\section{OCT Macular Map Reproducibility}

The 10 patients with repeat measurements had a median visual acuity of $0.52 \log$ MAR ranging from 0 to 1.3 ,
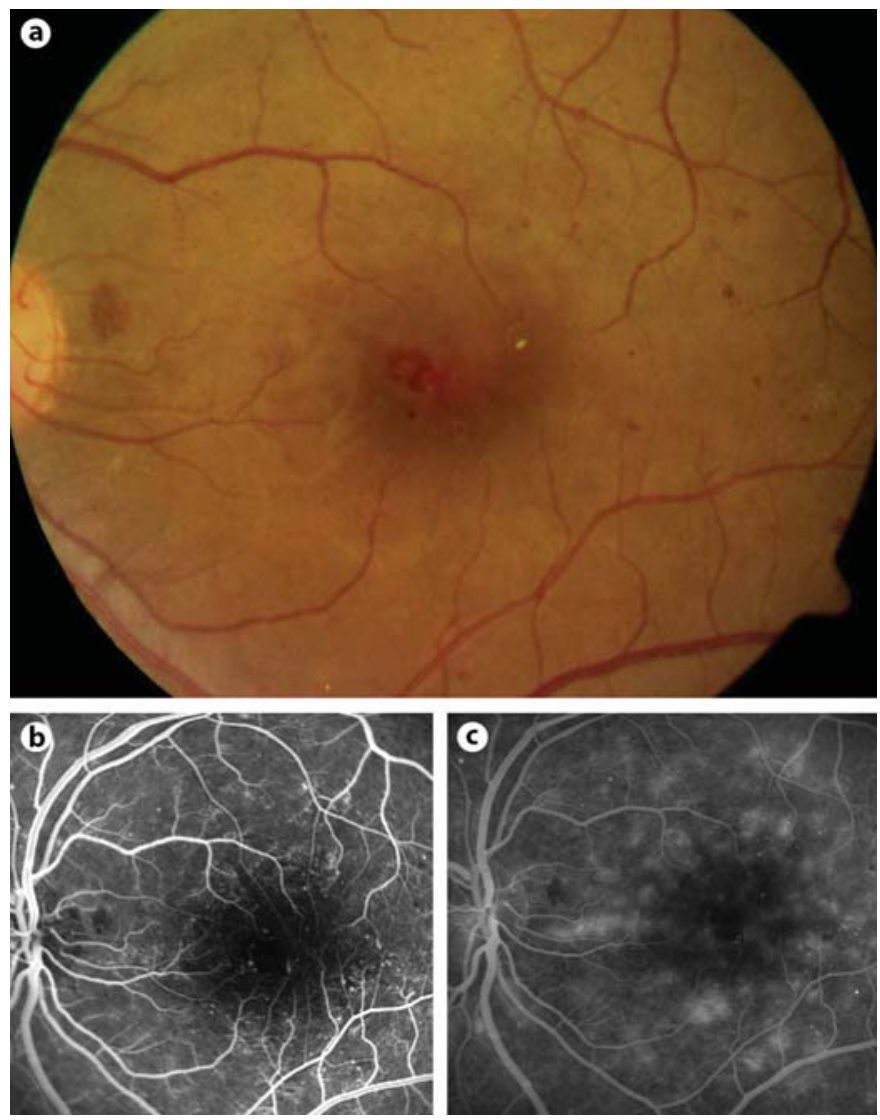

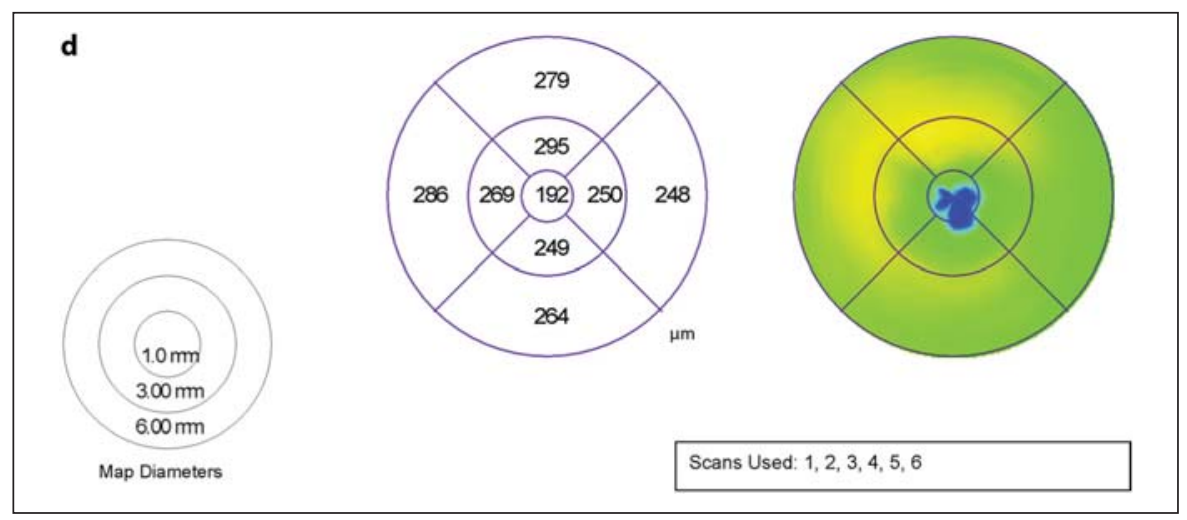

Fig. 1. Patient with diabetic macular edema. a Color fundus photograph of the left eye of a patient with mild clinically significant diabetic macular edema. b Early phase of fluorescein angiography. c Late phase of fluorescein angiography. d Corresponding regular macular thickness map computed from high-density scans $(1.28 \mathrm{~s} / \mathrm{scan})$. e Corresponding fast macular thickness map computed from lowdensity scans (0.32 s/scan).

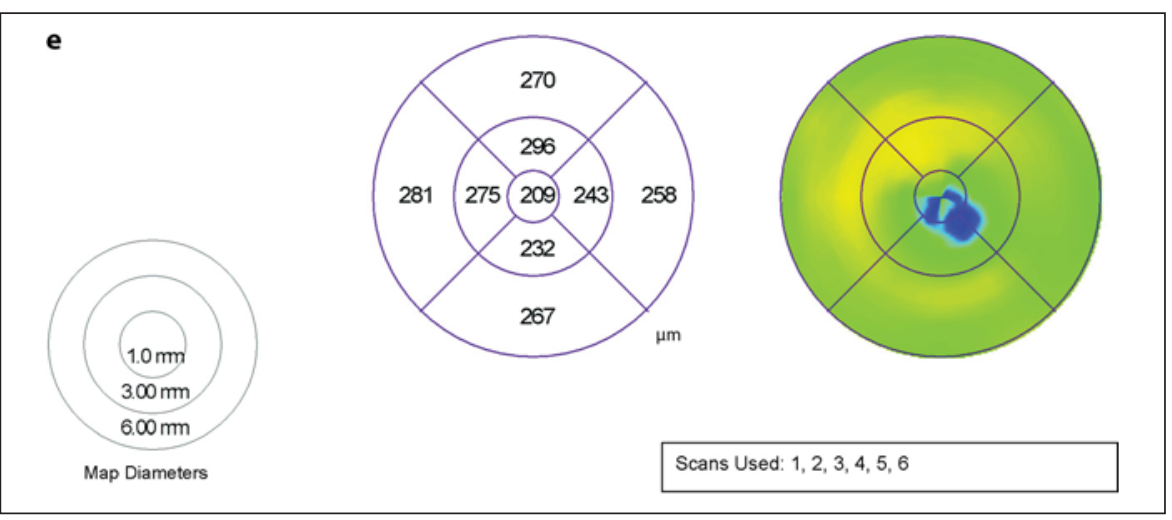


Table 2. Comparison of fast and regular OCT maps in diabetic macular edema

\begin{tabular}{llllll}
\hline OCT field & $\begin{array}{l}\text { Regular map } \\
\mu \mathrm{m}\end{array}$ & $\begin{array}{l}\text { Fast map } \\
\mu \mathrm{m}\end{array}$ & $\begin{array}{l}\text { Significance } \\
\text { of median } \\
\text { difference }\end{array}$ & $\begin{array}{l}\text { Correlation } \\
\text { of regular and } \\
\text { fast map values }\end{array}$ & $\begin{array}{l}\text { Significance of } \\
\text { correlation } \\
\mathrm{p}\end{array}$ \\
\hline 1 (center field) & $290 \pm 113$ & $287 \pm 97$ & n.s. & 0.95 & $<0.001$ \\
2 (central superior) & $315 \pm 105$ & $324 \pm 98$ & n.s. & 0.92 & $<0.001$ \\
3 (central temporal) & $314 \pm 97$ & $313 \pm 118$ & n.s. & 0.84 & $<0.001$ \\
4 (central inferior) & $317 \pm 97$ & $307 \pm 81$ & n.s. & 0.95 & $<0.001$ \\
5 (central nasal) & $295 \pm 90$ & $306 \pm 75$ & n.s. & 0.72 & $<0.001$ \\
6 (peripheral superior) & $284 \pm 109$ & $276 \pm 111$ & n.s. & 0.90 & $<0.001$ \\
7 (peripheral temporal) & $271 \pm 92$ & $283 \pm 106$ & n.s. & 0.88 & $<0.001$ \\
8 (peripheral inferior) & $272 \pm 77$ & $276 \pm 86$ & n.s. & 0.88 & $<0.001$ \\
9 (peripheral nasal) & $270 \pm 66$ & $275 \pm 69$ & n.s. & 0.85 & \\
\hline
\end{tabular}

Values are medians \pm interquartile ranges. n.s. $=$ Not significant.

Table 3. Reproducibility of fast and regular OCT maps in diabetic macular edema

\begin{tabular}{lllll}
\hline OCT field & $\begin{array}{l}\text { Fast maps } \\
\text { ICC }\end{array}$ & $\begin{array}{l}\text { Fast maps } \\
\text { intrasession SD, } \mu \mathrm{m}\end{array}$ & $\begin{array}{l}\text { Regular maps } \\
\text { ICC }\end{array}$ & $\begin{array}{l}\text { Regular maps } \\
\text { intrasession SD, } \mu \mathrm{m}\end{array}$ \\
\hline 1 (center field) & 0.97 & 10.71 & 0.99 & 7.23 \\
2 (central superior) & 0.93 & 10.63 & 0.95 & 9.43 \\
3 (central temporal) & 0.94 & 11.12 & 0.98 & 8.83 \\
4 (central inferior) & 0.93 & 8.32 & 0.90 & 13.23 \\
5 (central nasal) & 0.71 & 14.47 & 0.85 & 13.90 \\
6 (peripheral superior) & 0.70 & 13.85 & 0.88 & 8.49 \\
7 (peripheral temporal) & 0.84 & 15.93 & 0.97 & 9.97 \\
8 (peripheral inferior) & 0.95 & 6.47 & 0.91 & 8.44 \\
9 (peripheral nasal) & 0.71 & 16.13 & 0.96 & 5.69 \\
\hline
\end{tabular}

which corresponds to Snellen 20/50 with a range from $20 / 400$ to $20 / 20$. Table 3 gives the intrasession correlation coefficients (ICC) and the intrasession reproducibility for those patients. Very good ICCs between 0.70 and 0.99 are obtained, the corresponding intrasession standard deviations range between 6 and $16 \mu \mathrm{m}$. The session standard deviations did not correlate with any other factor included in the study, especially not with visual acuity.

\section{Discussion}

OCT has become a valuable imaging modality in the evaluation and management of various macular diseases, particularly with regard to diseases that result in abnormal macular thickness, such as macular edema. The Zeiss Stratus OCT allows not only good axial resolution of ap- proximately $10 \mu \mathrm{m}$ but also faster scanning than previous time domain models. This may be used for increasing the number of A scans as with the 'regular' macular map mode or allow faster scanning with a lower number of $\mathrm{A}$ scans as with the 'fast' macular thickness mode. Faster scanning should theoretically reduce motion artifacts and might therefore be beneficial in patients with reduced visual acuity. On the other hand due to less lateral resolution after the instrument's software processing the resulting thickness measurements may yield higher variability. The current study showed in patients with diabetic macular edema that no large differences exist between the 2 scanning modes. The variation of the OCT macular maps acquired in a longer time but with high lateral resolution was consistently lower (see table 3) than for the fast mode. Differences were, however, small. As absolute measurements did not differ between the 2 map- 
ping modes, this means that in practice both scanning modes can be interchanged. Still, the best reproducibility is obtained with higher sampling density even in patients with reduced visual acuity such as in diabetic macular edema.

These results are in concordance with a paper investigating reproducibility in normal subjects [17], where also good reproducibilites were found for both scanning modes with some advantage for the high-density scans. It is remarkable that the same is true for the patients with diabetic macular edema investigated in our study, although they have reduced visual acuity and ability to fixate. It appears important that in those patients the 2 scanning modes were applied in random order to avoid any bias, e.g. by getting tired. When comparing in detail the values reported for the normal subjects in Paunescu et al. [17], the diabetic patients in our study show between 2 and 3 times higher session standard deviations for the same 3 times repeated measurements. This is believed to be related to a reduced ability to fixate due to diabetic macular edema. Another possible explanation is that scan quality may be impaired by less fixation causing higher variance of scan analysis [18]. Interestingly, no correlation between OCT session variability and the patients' visual acuity could be shown in our study - fixation itself was not measured in this study. When transferring our results it should also be considered that the retinopathy level distribution in patients mostly represented more advanced retinopathy levels, while for instance in screening settings lower levels are to be expected. In comparison to the previous OCT 2000 version, where a reproducibility for macular thickness measurements of $<20$ $\mu \mathrm{m}$ was found in diabetic retinopathy [19], our current study observed a better reproducibility with the Stratus OCT. This may be related to the increased sampling density and axial resolution - and only to some minor degree to the higher speed of image acquisition with the Stratus OCT.

In summary, in patients with diabetic macular edema, fixation loss appears to be less of a concern, as image resolution is compromised in a fast macular scan protocol as compared to the regular, slower protocol. This regular scan protocol provides more data points and may therefore be more indicative of changes in the macula [20]. On the other hand differences in map repeatability are small and the absolute thickness values do not differ. Therefore the values of both protocols can be directly compared. This implies that in cases where regular scanning is difficult one can use the fast macular map, which yields similarly reliable results as the slower high-density mode. In summary, with the current study in a first attempt the comparability of slow and fast scanning has been shown in diabetic retinopathy. The results should be confirmed in a larger number of patients with time domain OCT and with other imaging technology applied in macular edema, such as spectral domain OCT.

\section{Acknowledgments}

The authors thank Mrs. S. Guthman and Mrs. N. Ehsani for expert technical assistance with fluorescein angiography and OCT scanning and help in the realization of this project.

L.C. and A.S.N. had full access to all the data in the study and take responsibility for the integrity of the data and the accuracy of the data analysis.

\section{References}

1 Stefansson E, Bek T, Porta M, Larsen N, Kristinsson JK, Agardh E: Screening and prevention of diabetic blindness. Acta Ophthalmol Scand 2000;78:374-385.

2 Klein R, Klein BE, Moss SE: Visual impairment in diabetes. Ophthalmology 1984;91: $1-9$.

3 Early Treatment Diabetic Retinopathy Study Research Group: Photocoagulation for diabetic macular edema: Early Treatment Diabetic Retinopathy Study report number 1 . Arch Ophthalmol 1985;103:1796-1806.

4 Early Treatment Diabetic Retinopathy Study Research Group: Focal photocoagulation treatment of diabetic macular edema - Relationship of treatment effect to fluorescein angiographic and other retinal characteris- tics at baseline: ETDRS report No 19. Arch Ophthalmol 1995;113:1144-1155.

5 Haritoglou C, Kook D, Neubauer AS, Wolf AH, Priglinger S, Strauss RW, Gandorfer A, Ulbig M, Kampik A: Intravitreal bevacizumab (Avastin) therapy for persistent diffuse diabetic macular edema. Retina 2006;26: 999-1005.

6 Aiello LP, Clermont A, Arora V, Davis MD, Sheetz MJ, Bursell SE: Inhibition of PKC beta by oral administration of ruboxistaurin is well tolerated and ameliorates diabetes-induced retinal hemodynamic abnormalities in patients. Invest Ophthalmol Vis Sci 2006; 47:86-92.

7 Kinyoun J, Barton F, Fisher M, Hubbard L, Aiello L, Ferris F 3rd; ETDRS Research
Group: Detection of diabetic macular edema: ophthalmoscopy versus photography Early Treatment Diabetic Retinopathy Study Report Number 5. Ophthalmology 1989;96: 746-750; discussion 750-741.

8 Neubauer AS, Priglinger S, Ullrich S, Bechmann M, Thiel MJ, Ulbig MW, Kampik A: Comparison of foveal thickness measured with the retinal thickness analyzer and optical coherence tomography. Retina 2001;21: 596-601.

9 Neubauer AS, Priglinger S, Thiel MJ, Bechmann M, Ulbig MW: Retinal maps: retinal thickness analyzer (RTA) compared to optical coherence tomography (OCT). IOVS Suppl 2001;42:S793. 
10 Polito A, Shah SM, Haller JA, Zimmer-Galler I, Zeimer R, Campochiaro PA, Vitale S: Comparison between retinal thickness analyzer and optical coherence tomography for assessment of foveal thickness in eyes with macular disease. Am J Ophthalmol 2002; 134:240-251.

11 Shahidi M, Ogura Y, Blair NP, Rusin MM, Zeimer R: Retinal thickness analysis for quantitative assessment of diabetic macular edema. Arch Ophthalmol 1991;109:11151119.

12 Brown JC, Solomon SD, Bressler SB, Schachat AP, DiBernardo C, Bressler NM: Detection of diabetic foveal edema: contact lens biomicroscopy compared with optical coherence tomography. Arch Ophthalmol 2004;122:330-335.
13 Pires I, Bernardes RC, Lobo CL, Soares MA, Cunha-Vaz JG: Retinal thickness in eyes with mild nonproliferative retinopathy in patients with type 2 diabetes mellitus: comparison of measurements obtained by retinal thickness analysis and optical coherence tomography. Arch Ophthalmol 2002;120: 1301-1306.

14 Early Treatment Diabetic Retinopathy Study Research Group: Grading diabetic retinopathy from stereoscopic color fundus photographs - an extension of the modified Airlie House classification: ETDRS report number 10. Ophthalmology 1991;98:786-806.

15 Hee MR, Izatt JA, Swanson EA, Huang D, Schuman JS, Lin CP, Puliafito CA, Fujimoto JG: Optical coherence tomography of the human retina. Arch Ophthalmol 1995;113: 325-332.

16 Huang D, Swanson EA, Lin CP, Schuman JS Stinson WG, Chang W, Hee MR, Flotte T, Gregory K, Puliafito CA, et al: Optical coherence tomography. Science 1991;254:11781181.
17 Paunescu LA, Schuman JS, Price LL, Stark PC, Beaton S, Ishikawa H, Wollstein G, Fujimoto JG: Reproducibility of nerve fiber thickness, macular thickness, and optic nerve head measurements using StratusOCT. Invest Ophthalmol Vis Sci 2004;45: 1716-1724.

18 Strauss RW, Scholz F, Ulbig MW, Kampik A, Neubauer AS: Artifacts in optical coherence tomography (OCT) imaging of the retina (in German). Klin Monatsbl Augenheilkd 2007; 224:47-51.

19 Massin P, Vicaut E, Haouchine B, Erginay A, Paques M, Gaudric A: Reproducibility of retinal mapping using optical coherence tomography. Arch Ophthalmol 2001;119: 1135-1142.

20 Chan A, Duker JS: A standardized method for reporting changes in macular thickening using optical coherence tomography. Arch Ophthalmol 2005;123:939-943. 